\title{
More Qualitative Cosmology
}

\author{
C. B. Collins \\ Department of Applied Mathematics and Theoretical Physics \\ Silver Street, Cambridge, England
}

Received July 14, 1971

\begin{abstract}
Standard geometric techniques of differential equation theory are employed to determine the qualitative behaviour of a set of non-rotating perfect-fluid cosmologies, whose spatially homogeneous hypersurfaces admit a 3-parameter group of isometries of Bianchi types I, II, III, V, or VI. In this way we are led to some new exact solutions of the field equations.

The field equations for a broad class of cosmological models are presented in a regularised form, limitations on the use of this procedure are examined, and some suggestions are made of ways of avoiding the difficulties that arise.
\end{abstract}

\section{Qualitative Methods}

It may be readily shown [5] that, for a certain class of perfect-fluid non-rotating and spatially homogeneous (but usually anisotropic) cosmological models, the Einstein field equations can be written in the form

$$
\begin{aligned}
& \frac{d x}{d \Omega}=x\left\{(3 \gamma-2)(1-x)-\beta^{\prime 2}+\frac{2}{3} \Lambda z\right\} \\
& \frac{d z}{d \Omega}=-2 z\left\{1+\frac{1}{2}(3 \gamma-2) x+\frac{1}{2} \beta^{\prime 2}-\frac{1}{3} \Lambda z\right\} \\
& \frac{d \beta_{1}}{d \Omega}=\beta_{1}^{\prime} \\
& \frac{d \beta_{2}}{d \Omega}=\beta_{2}^{\prime} \\
& \frac{d \beta_{1}^{\prime}}{d \Omega}=\frac{1}{2} \beta_{1}^{\prime}\left\{4-(3 \gamma-2) x-\beta^{\prime 2}+\frac{2}{3} \Lambda z\right\}-\frac{1}{2} z e^{2 \Omega} \frac{\partial V_{1}}{\partial \beta_{1}}
\end{aligned}
$$

and

$$
\frac{d \beta_{2}^{\prime}}{d \Omega}=\frac{1}{2} \beta_{2}^{\prime}\left\{4-(3 \gamma-2) x-\beta^{\prime 2}+\frac{2}{3} \Lambda z\right\}-\frac{1}{2} z e^{2 \Omega} \frac{\partial V_{1}}{\partial \beta_{2}},
$$


with the first integral

$$
{\beta^{\prime 2}}^{2}=4-4 x-\frac{4}{3} \Lambda z-z e^{2 \Omega}\left(V_{1}-\delta_{\mathrm{IX}}^{T}\right) .
$$

In this system of equations, $\beta_{1}^{\prime}$ and $\beta_{2}^{\prime}$ are quantities which measure the rate of shear (i.e. anisotropy) in terms of the expansion, $\theta$, of the models, ${\beta^{\prime}}^{2} \equiv{\beta_{1}^{\prime 2}}^{2}+{\beta_{2}^{\prime 2}}_{2}, z=9 \theta^{-2}, x \equiv \frac{1}{3} \mu z$ (where $\mu$ is the total energy density) measures the dynamical importance of the matter content and $V_{1} \equiv V_{1}\left(\beta_{1}, \beta_{2}\right)=\delta_{\mathrm{IX}}^{T}-\frac{2}{3} R^{*} e^{-2 \Omega}$ is an effective potential for the model's anisotropy, $R^{*}$ being the Ricci curvature of the hypersurfaces of homogeneity [20]. A barotropic equation of state of the form $p=(\gamma-1) \mu$ has been assumed, where $p$ is the pressure and $\gamma$ is a constant satisfying $1 \leqq \gamma \leqq 2$. This inequality is necessary for the existence of local mechanical stability and for the speed of sound in the fluid to be no greater than the speed of light [8]. Whether or not matter exists with the limiting "stiff" equation of state $p=\mu$ is a controversial question $([11,39]$, cf. [30]); many authors prefer to assume $1 \leqq \gamma \leqq \frac{4}{3}$. It will be found later that for each model considered, the nature of many solutions is qualitatively the same for all values of $\gamma$ satisfying $1 \leqq \gamma<2$, but that the case $\gamma=2$ leads to a highly exceptional behaviour. This statement can be reformulated in the context of the theory of differential equations: the system (1.1)-(1.5) is structurally stable against variations of $\gamma(1 \leqq \gamma<2)$.

The equations of our system have been expressed in terms of derivatives with respect to a new time-variable, $\Omega$, which can always be usefully defined for expanding models by the equation $\ell=e^{-\Omega}$, where $\ell$ is the "representative length scale", or "radius of the universe". Misner [23-26] first used this $\Omega$-time with the variable $\boldsymbol{\beta} \equiv\left(\beta_{1}, \beta_{2}\right)$ to formulate the field equations for models of Bianchi types I and IX. MacCallum [20] (c.f. Jacobs and Hughston [13]) has generalized this work to an entire family of models: in the notation of Ellis and MacCallum [9] this family consists of all Class $A$ models, and the set of Class $B$ with $n_{\alpha}^{\alpha}=0(\alpha=1,2,3)$. It is this family to which we will later restrict our attention. The remaining set of models, Class B with $n_{\alpha}^{\alpha} \neq 0$, is highly intractable. In Class B with $n^{\alpha}{ }_{\alpha}=0$, the Eqs. (1.5) are modified, because $\sqrt{3} \beta_{1}=k \beta_{2}$ for some constant $k$. If $\beta$ be defined by $-\beta \sqrt{\left(3+k^{2}\right)}$ $\equiv k \beta_{1}+\sqrt{3} \beta_{2}$, we obtain the equation

$$
\frac{d \beta^{\prime}}{d \Omega}=\frac{1}{2} \beta^{\prime}\left\{4-(3 \gamma-2) x-{\beta^{\prime 2}}^{2}+\frac{2}{3} \Lambda z\right\}-\frac{1}{2} z e^{2 \Omega} \frac{\partial V}{\partial \beta},
$$

in which $V_{1}$ is replaced by $V \equiv V_{1}+V_{2} e^{4 \Omega}$, where $V_{2}=\frac{4}{3} b^{2} e^{2 \sqrt{3} \beta}$ and $b=0$ except in case Bbii (see [20]).

The equations have now been "regularised", in the sense that the right-hand sides of (1.1)-(1.5) are analytic functions of quantities whose 
derivatives appear on the left, although they do explicitly contain the time-variable, $\Omega$, and so form a "non-autonomous system". They may be regularised in the sense of Oszvath [29], i.e. regularised to form an analytic autonomous system, either by adjoining a further equation (e.g. $\frac{d \Omega}{d \Omega}=1$; cf. [34]) to the system, or by using the first integral Eq. (1.6) to eliminate the time-dependence, or by a further change of variable. The first method, while formally valid, is of little practical consequence. The second method, elimination of $\Omega$, renders the system autonomous, but generally non-analytic, although some exceptional cases can be studied (see Section 2). Oszvath's scheme, which uses new variables, unfortunately seems to necessitate an increase in the number of equations: Oszvath (op. cit.) has examined axisymmetric $\left(\beta_{2} \equiv 0\right)$ dustfilled $(\gamma=1)$ non-rotating Bianchi types I, II, VIII and IX with $\Lambda=0$, and has obtained a system of equations which is analytic, autonomous and with the additional property that the functions appearing on the righthand sides are bounded for finite values of the variables. This representation is analogous to the regularisation procedure (which appears to have been initiated by Levi-Civita $[17,18]$ ) used in the three-body problem of classical mechanics. For instance, Waldvogel [38] and Arenstorf [1] study the elliptic restricted three-body problem in three dimensions: that is, the motion in $\mathscr{R}^{3}$ of a mass point $P$ in the Newtonian gravitational field of two other mass points, $P_{1}$ and $P_{2}$, which move in elliptic orbits about their centre of mass, with $P$ having negligible effect on this motion. The aim is to find suitable transformations of the distance-, velocity-, and time-variables which yield differential equations whose right-hand sides are holomorphic on that part of phase-space containing all trajectories of $P$ (whereas in the original notation, infinite values of the potential would occur at a collision of $P$ with $P_{1}$ or $P_{2}$ ). A new timevariable is defined which corresponds to Oszvath's transformation $t \rightarrow \tau$ :

$$
\tau=\int_{t_{0}}^{t} \frac{d t}{B}
$$

(where $B$ is a representative length) and the existence of a "collision path" to $P_{1}$ or $P_{2}$ (corresponding to $B \rightarrow 0$ as $t \rightarrow t^{*}+0$ ) can be demonstrated. Further, the "Sundman integral" (1.7) converges along such a path (i.e. $t^{*}>-\infty$ ). The advantage of regularisation is now apparent: the behaviour of the new dependent variables at a collision is known (they all tend to finite limits) and so the solution is holomorphic in some neighbourhood of the collision, i.e. a Taylor series expansion exists (with some non-zero radius of convergence) for the new variables in positive integral powers of $\tau-\tau^{*}$, where $\tau^{*}=\tau\left(t^{*}\right)$. On transforming back to the original coordinates, we obtain a series in powers of $t-t^{*}$, the 
dominant term of which may involve fractional or inverse powers, or both. Knowledge of the motion "through" the collision is obtained, and any further collisions can be treated similarly.

The situation in cosmology appears to be somewhat more complicated. Firstly, we must examine the Sundman integral, and possibly make a further change of variable to ensure its convergence (this question is related to that of the existence of particle horizons, cf. [19]). Secondly, the behaviour of our dependent variables at the singularity does not in general seem to be immediately evident (thus the solutions do not appear to be necessarily holomorphic at the singularity: for instance, Oszvath's variables $x, y, z$ and $B$ may a priori become infinite, or oscillate infinitely often on approaching the singularity). We should like a change of variables so that the field equations are of the form $\frac{d x}{d T}=\boldsymbol{X}(\boldsymbol{x})$, where $\boldsymbol{X}(\boldsymbol{x})$ is analytic and bounded for all finite $\boldsymbol{x}=\left(x_{1}, x_{2}, \ldots, x_{m}\right) \in \mathscr{R}^{m}$, with the additional conditions $x \rightarrow x_{0}$ as $T \rightarrow T_{0}$, where $T_{0}$ is a finite $T$-time at which the singularity occurs and $x_{0}$ is finite. If this cannot be done we could seek the existence of a theorem giving conditions under which "singularities" (strictly, points beyond which analytic continuation is impossible) do or do not occur in a finite time in normal algebraic autonomous systems (i.e., in systems of the form $\frac{d \boldsymbol{x}}{d t}=\boldsymbol{P}(\boldsymbol{x})$, where $\boldsymbol{P}$ is a vector with $m$ polynomial components), and if they occur in a finite time, some information on the manner in which they do so (cf. the work of Boutroux [3] and Hardy [10], who have examined the behaviour of solutions of a single polynomial equation $(m=1)$; it is immediately clear from phase plane methods such as those described in this section that when $m=1$ any singularity necessarily occurs within a finite time whenever the degree of $\boldsymbol{P}$ is greater than 1, and that the singularity behaves as an inverse fractional power of the time variable). It is possible that this result could be extended to more general systems, but so far the problem appears to have received little attention (cf. [21]).

Oszvath's regularisation can be readily extended to models which contain a perfect fluid with an equation of state $p=(\gamma-1) \mu(1 \leqq \gamma \leqq 2)$ and which are of Class $A$ or Class $B\left(n^{\alpha}{ }_{\alpha}=0\right.$, not $\left.B b i i\right)$. The equations for these models are given in [9] (Eqs. (4.9) and (6.7)), and with the change of variables $x=\frac{X}{(Y Z)^{1 / 2}}, y=-\frac{2}{x^{2}}\left(\frac{Y^{\prime}}{Y}+\frac{Z^{\prime}}{Z}\right), z=-\frac{4}{x}\left\{\frac{X^{\prime}}{X}+\frac{1}{2}\left(\frac{Y^{\prime}}{Y}+\frac{Z^{\prime}}{Z}\right)\right\}$, $q=\frac{1}{x^{2}}\left(\frac{Y^{\prime}}{Y}-\frac{Z^{\prime}}{Z}\right), d \bar{\tau}=\frac{d \tau}{x^{2}}=\frac{(Y Z)^{1 / 2} d t}{X^{2}}$, and in Class $\mathrm{A}$ with $r=\frac{N_{2} Y}{Z}-\frac{N_{3} Z}{Y}, s=\frac{N_{2} Y}{Z}+\frac{N_{3} Z}{Y}$, we can obtain regularised equa- 
tions for both classes. For Class A models we have the system

$$
\begin{aligned}
x^{\prime}= & \frac{1}{2} x^{3} y-\frac{1}{4} x^{2} z, \\
y^{\prime}= & \frac{1}{4}(\gamma-1) x y z+\frac{1}{8}(2-\gamma) x^{2} y^{2}+\frac{1}{2}(2-\gamma) x^{2} q^{2} \\
& +\gamma N_{1} x^{2} s-\frac{1}{2}(2+\gamma) N_{1}^{2} x^{4}+\frac{1}{2}(2-\gamma) r^{2}, \\
z^{\prime}= & \frac{1}{2}(\gamma-1) x^{2} y z+\frac{1}{4}(2-\gamma) x^{3} y^{2}+(2-\gamma) x^{3} q^{2} \\
& +(2-\gamma) N_{1}^{2} x^{5}+2(\gamma-1) N_{1} x^{3} s-\gamma x r^{2}, \\
q^{\prime}= & \frac{1}{4} x z q-r s+N_{1} x^{2} r, \\
r^{\prime}= & x^{2} q s,
\end{aligned}
$$

and

$$
s^{\prime}=x^{2} q r,
$$

with the "first integrals"

$$
s^{2}-r^{2}=4 N_{2} N_{3}
$$

and

$$
\frac{4 M}{X^{\gamma-1}(Y Z)^{\gamma-1 / 2}}=\frac{1}{2} y z-\frac{1}{4} x y^{2}-x q^{2}+2 N_{1} x s-\frac{r^{2}}{x}-N_{1}^{2} x^{3},
$$

while for Class B $\left(n_{\alpha}^{\alpha}=0\right.$, not Bbii) models,

$$
\begin{aligned}
x^{\prime}= & \frac{1}{2} x^{3} y-\frac{1}{4} x^{2} z, \\
y^{\prime}= & \frac{1}{4}(\gamma-1) x y z+\frac{1}{8}(2-\gamma) x^{2} y^{2}+\frac{1}{2}(2-\gamma) x^{2} q^{2} \\
& +2\left[(2-\gamma) q_{0}{ }^{2}-(3 \gamma-2) a_{0}{ }^{2}\right], \\
z^{\prime}= & \frac{1}{2}(\gamma-1) x^{2} y z+\frac{1}{4}(2-\gamma) x^{3} y^{2}+(2-\gamma) x^{3} q^{2} \\
& -4 x\left[\gamma q_{0}{ }^{2}+(3 \gamma-2) a_{0}^{2}\right],
\end{aligned}
$$

and

$$
q^{\prime}=\frac{1}{4} x z q+4 a_{0} q_{0}
$$

with the "first integrals"

$$
y-\frac{1}{2} \frac{z}{x}=\frac{q_{0}}{a_{0}} q
$$

and

$$
\frac{4 M}{X^{\gamma-1}(Y Z)^{\gamma-1 / 2}}=\frac{1}{2} y z-\frac{1}{4} x y^{2}-x q^{2}-\frac{4}{x}\left(3 a_{0}{ }^{2}+q_{0}{ }^{2}\right) .
$$


In the above, a dash $\left(^{\prime}\right)$ denotes differentiation with respect to $\bar{\tau}$, the notation and convention of [20] are employed (thus $q_{0}$ is not directly related to the variable $q$ ), and the phrase "first integral" is used rather imprecisely, since in each pair of such equations, the second involves quantities not expressed in terms of the variables of the system to which it belongs. The systems hold for zero cosmological constant, $\Lambda$, no progress having been made in attempts to find a regularized system either when $\Lambda \neq 0$ or when models of Class B $\left(n_{\alpha}^{\alpha} \neq 0\right.$ or $\left.B b i i\right)$ are considered. For models of Class A with $q \equiv r \equiv 0, \gamma=1$ and $Y \equiv Z$, we obtain Oszvath's system [29], with $\frac{d}{d \bar{\tau}}=x^{2} \frac{d}{d \tau}$.

Of course, this is not the only possible regularisation. For instance, we find that for all ${ }^{1}$ axisymmetric dust models $(Y \equiv Z, \gamma=1)$ of Class A, the transformations $\bar{x}=x / Y, \bar{y}=y / Y, \bar{z}=z / Y^{2}, \bar{s}=s / Y^{2}$ give the regularised system

and

$$
\begin{aligned}
& \dot{\bar{x}}=\frac{3}{4} \bar{x} \bar{y}-\frac{1}{4} \bar{z}, \\
& \dot{\bar{y}}=\frac{3}{8} \bar{y}^{2}+N_{1} \bar{s}-\frac{3}{2} N_{1}^{2} \bar{x}^{2}, \\
& \dot{\bar{z}}=\frac{1}{2} \bar{x} \bar{y}^{2}+N_{1} \bar{x}^{3}+\frac{1}{2} \bar{y} \bar{z},
\end{aligned}
$$

$$
\dot{\bar{S}}=\frac{1}{2} \bar{y} \bar{s}
$$

with "first integrals"

$$
\bar{s}=\left(N_{2}+N_{3}\right) / Y^{2}
$$

and

$$
\frac{4 M}{Y^{4}}=\frac{1}{2} \bar{y} \bar{z}-\frac{1}{4} \bar{x} \bar{y}^{2}+2 N_{1} \bar{x} \bar{s}-N_{1}^{2} \bar{x}^{3},
$$

where a dot $\left({ }^{\prime}\right)$ denotes differentiation with respect to the original timevariable, $t$. This might be preferable to the previous regularisation, because there is now no need to consider the question of convergence of the Sundman integral.

It may be advantageous to study the field equations in the form of an autonomous analytic system, because they are then more amenable to the application both of numerical methods and of qualitative methods in the theory of differential equations. We shall use the latter approach. The qualitative theory is well understood for autonomous systems of two first-order equations, for in this case the types of "singular-points" (where the right-hand sides vanish simultaneously) are rather limited, and the various behaviours possible may be readily classified (see e.g.

${ }^{1}$ The type VII $_{0}$ model of this set admits a group of type I [9], and is here considered as such. 
$[27,32,37])$. The entire qualitative features of the solutions may then be mapped out by trajectories in a two-dimensional phase-space (giving rise to the phrase "plane autonomous systems"), provided that this space does not have the global topology of the torus $\mathscr{S}^{1} \times \mathscr{S}^{1}$, i.e. provided that the two unknown variables do not both behave as angles. Much difficulty is encountered in attempting to extend the general theory to more complicated systems, owing to the great variety of possibilities in the types of singular points, and to the fact that the Jordan curve theory, on which a considerable part of the plane theory depends, has no direct analogue in higher dimensions: intersection properties of curves in three dimensions are greatly different from those in two. Numerical or computer methods will generally require the system to be bounded in the region of $(x, t)$ space considered, where $x$ refers to the dependent variables and $t$ the independent variable in the system of equations whose solutions are being investigated. Consequently the singularity $\ell=0$ cannot be examined by such methods using the $\beta-\Omega$ notation $(\Omega \rightarrow+\infty)$, although attempts at discovering the behaviour up to finite $\Omega$-values have been made (see e.g. [4, 22, 28, 31]).

Although Oszvath's system and its above extension may have all the properties required for computational purpose, it seems that there is no immediate subsystem which also has these properties, and which lends itself to other methods of analysis. For this reason, we consider henceforth the system of equations in the form (1.1)-(1.5), bearing in mind that the method of regularisation may yet yield further systems amenable to geometric study.

\section{A Tractable Subsystem}

We will now investigate the possibility of obtaining autonomous analytic subsystems from Eqs. (1.1)-(1.6), with $\Lambda=0$. The form of the potential $V_{1}$ will be taken from the results quoted in [20]. In the case of Class B with $n_{\alpha}^{\alpha}=0$ (types $\mathrm{V}$ and $\mathrm{VI}_{h}, h \neq-\frac{1}{9}$ ), (1.1) and (1.5b) give such a subsystem, because $V_{1}$ is a purely exponential function of $\beta$. (1.6) can then be used to eliminate $z e^{2 \Omega}$, and a plane autonomous system, $\left[x, \beta^{\prime}\right]$, results for the variables $x$ and $\beta^{\prime}$. For models of Class $\mathrm{A}$, we can form a subsystem $\left[x, \beta_{1}^{\prime}\right]$ of the required type whenever $\beta_{2}^{\prime} \equiv 0$ (which corresponds to axisymmetry), and $V_{1}$ is purely exponential (types I, II, $\mathrm{VI}_{0}$ and $\mathrm{VII}_{0}$ ), by considering (1.1) and (1.5) together and eliminating $z e^{2 \Omega}$ by means of (1.6). Other special subsystems which also yield to qualitative analysis include the general type II models with $\Lambda=0$, and the type I model with $\Lambda \neq 0$. These models are discussed in Section 3 .

For models of type I $\left(V_{1} \equiv 0\right)$, the motion is always rectilinear in the $\beta$-plane, even when $\Lambda \neq 0$, as can be seen from (1.5a). Thus $\beta_{1}^{\prime}$ and $\beta_{2}^{\prime}$ 
are proportional: either $\beta_{1}^{\prime} \equiv 0$ or $\beta_{2}^{\prime} \equiv \lambda \beta_{1}^{\prime}$ for some constant $\lambda$. If $\Lambda=0$, we obtain from (1.1), (1.5a) and (1.6) a system of two independent equations which can be solved exactly (see e.g. [12] and references therein), or can be treated qualitatively in a manner depicted in Fig. 1 (the $\Lambda=0$ solutions are depicted on the curved part of the boundary of the D-shaped region), from which we can read off the pattern of the behaviour of the solutions ${ }^{2}$. The diagrams are drawn in $\Omega$-time, that is, arrows indicate the behaviour approaching the singularity (increasing $\Omega$, decreasing $t$ ), and $\bar{\beta}^{\prime}=\beta_{2}^{\prime}$ or $\beta_{1}^{\prime}$ according as $\beta_{1}^{\prime} \equiv 0$ or $\beta_{2}^{\prime} \equiv \lambda \beta_{1}^{\prime}$. From (1.5a), $\bar{\beta}^{\prime \prime}$ has the same sign as $\bar{\beta}^{\prime}$ for $\gamma \neq 2$, and $\bar{\beta}^{\prime \prime} \equiv 0$ when $\gamma=2$. From Fig. 1 we see that for $1 \leqq \gamma<2$ there are certain special cases: the Robertson-Walker solution for spatial hypersurfaces of homogeneity with zero curvature $\left(x \equiv 1, \bar{\beta}^{\prime} \equiv 0\right)$, and two empty solutions with $\bar{\beta}^{\prime}= \pm d[36]$. It can be seen that there are no "bounces": $\bar{\beta}^{\prime}$ is of constant sign $[20,26]$. Matter effects are generally unimportant in the early stages (in that $x \rightarrow 0$ as $\Omega \rightarrow+\infty$ ), the only exception being the Robertson-Walker solution. The models "isotropize" away from the singularity, that is $\beta^{\prime} \propto \sigma / \theta \rightarrow 0$ as $\Omega \rightarrow-\infty$ (cf. [20]). When $\gamma=2, x$ and $\bar{\beta}^{\prime}$ are identically constant. Solutions have been obtained in closed form by, for example, Jacobs [12]. They do not isotropize, and matter effects are important at all times for all such models. Again, there is no change of direction of motion in the $\beta$-plane.

We can examine the nature of the types of singularities that can occur by using the information contained in the diagrams. For type I models, the question has been extensively investigated $[12,20]$; in general, there are pancake or cigar singularities with the 1-, 2- or 3-axes distinguished. The only exceptional case is the Robertson-Walker model, which has a point singularity.

For the remaining models dealt with in this section, $V_{1}$ is purely exponential, i.e. $V_{1}=D e^{C \beta}$ for constants $D \neq 0$ and $C$, and for $\beta=\beta_{1}$ in Class $\mathrm{A}$ and $\beta=-\frac{k \beta_{1}+\sqrt{3} \beta_{2}}{V\left(3+k^{2}\right)}$ in Class B $\left(n_{\alpha}^{\alpha}=0\right)$. Eqs. (1.1), (1.5) and (1.6) now give the system

$$
\frac{d x}{d \Omega}=x\left\{(3 \gamma-2)(1-x)-{\beta^{\prime 2}}^{2} \quad \equiv X\left(x, \beta^{\prime}\right)\right.
$$

${ }^{2}$ Figs. 1-7 are drawn schematically: for instance, no distinction is made in Fig. 5(ai) between the general behaviour and the case in which $2 C=3 \gamma-2$, where a "star-like" node occurs at $(0,2)$, i.e. trajectories approach this point from all possible directions, and not just along the boundary. The boundary of the $D$-shaped region in Figs. 2-5 intersects the line $x=0$ at $\beta^{\prime}$-values \pm 2 , whereas in Fig. 1 the appropriate values are $\pm d$, where $d=2$ for $\beta_{1}^{\prime} \equiv 0$ and $d=2\left(1+\lambda^{2}\right)^{-1 / 2}$ for $\beta_{2}^{\prime} \equiv \lambda \beta_{1}^{\prime}$. In Fig. 7, the appropriate $\beta^{\prime}$-values are $\pm c$, where $c=\left[\frac{3}{16}(2+\gamma)(10-3 \gamma)\right]^{1 / 2}$. 
and

$$
\frac{d \beta^{\prime}}{d \Omega}=\frac{1}{2} \beta^{\prime}\left\{4-(3 \gamma-2) x-{\beta^{\prime 2}}^{2}\right\}-\frac{1}{2} C\left(4-4 x-{\beta^{\prime 2}}^{2}\right) \equiv B\left(x, \beta^{\prime}\right) .
$$

This pair of equations is now analysed using qualitative methods, with the values of $C$ taken as $4,-2,0$ and $2 k / V\left(3+k^{2}\right)$ for types II, $\mathrm{VI}_{0}, \mathrm{~V}$ and $\mathrm{VI}_{h}$ respectively. The case $\mathrm{VII}_{0}$ with $\beta_{2} \equiv 0$ admits a group of type I [9], and so has already been discussed; it is "locally rotationally symmetric" (LRS), in the sense of Ellis [7] and of Stewart and Ellis [33]. For each value of $C$, we proceed by calculating the singular points and then use standard techniques to evaluate their nature. Because of (1.6) we need consider only the region $\beta^{\prime 2}+4 x \leqq 4, x \geqq 0$. The results of the qualitative analysis are depicted schematically in Figs. $2-5$. In the type II, $\mathrm{VI}_{0}$ and $\mathrm{VI}_{h}$ models for $1 \leqq \gamma<2$ a "focus" can occur: there is some point in the region under consideration out of which trajectories spiral infinitely many times. In such cases, "limit cycles", or closed trajectories, can possibly occur, corresponding to periodic solutions. There are certain theorems which give conditions for the existence or non-existence of such behaviour. We have used a theorem due to Dulac [6], which generalises a result of Bendixson [2]: we exhibit the function $f\left(x, \beta^{\prime}\right)=x^{-3 / 2}\left(4-4 x-\beta^{\prime 2}\right)^{-1}$ which, on the singly-connected region $\beta^{\prime 2}+4 x<4, x>0$ of the $x-\beta^{\prime}$ phase plane is continuous with continuous derivatives, and satisfies $\frac{\partial}{\partial x}(f X)+\frac{\partial}{\partial \beta^{\prime}}(f B)>0$. It follows that there can be no closed trajectories, and that the motion is as shown in our diagrams. We now give a brief discussion of the main properties relating to these diagrams.

For type II models the typical behaviour is that the model "starts" $(\Omega \rightarrow-\infty)$ at a non-zero value of $\beta_{1}^{\prime}$ (and so does not isotropize in $t$-time), executes one bounce (where $\beta_{1}^{\prime}=0$ ) and as $\Omega \rightarrow+\infty, \beta_{1}^{\prime} \rightarrow-2$ and $x \rightarrow 0$, indicating that matter effects are negligible near the singularity (which, in this general case, is a pancake with the 1-axis distinguished [20]). This behaviour agrees with that predicted in [13] and [20], where the analyses were based on the concept of a receding potential wall (described by the function $V$ ) which in type II is parallel to the $\beta_{2}$ axis: the universepoint bounces exactly once off this wall, and "ultimately" $(\Omega \rightarrow+\infty) \beta_{1}^{\prime}$ is negative. However, it is shown in Fig. 2 that certain exceptional cases arise, whose anomalous behaviour is not accounted for in the previous works. For $1 \leqq \gamma<2$, there is one solution for which $x$ and $\beta_{1}^{\prime}$ are identically constant, and another for which $x \rightarrow 1$ and $\beta^{\prime} \rightarrow 0$ on approaching the singularity. Both solutions have point singularities. The former is given in closed form in the appendix, and has already been 

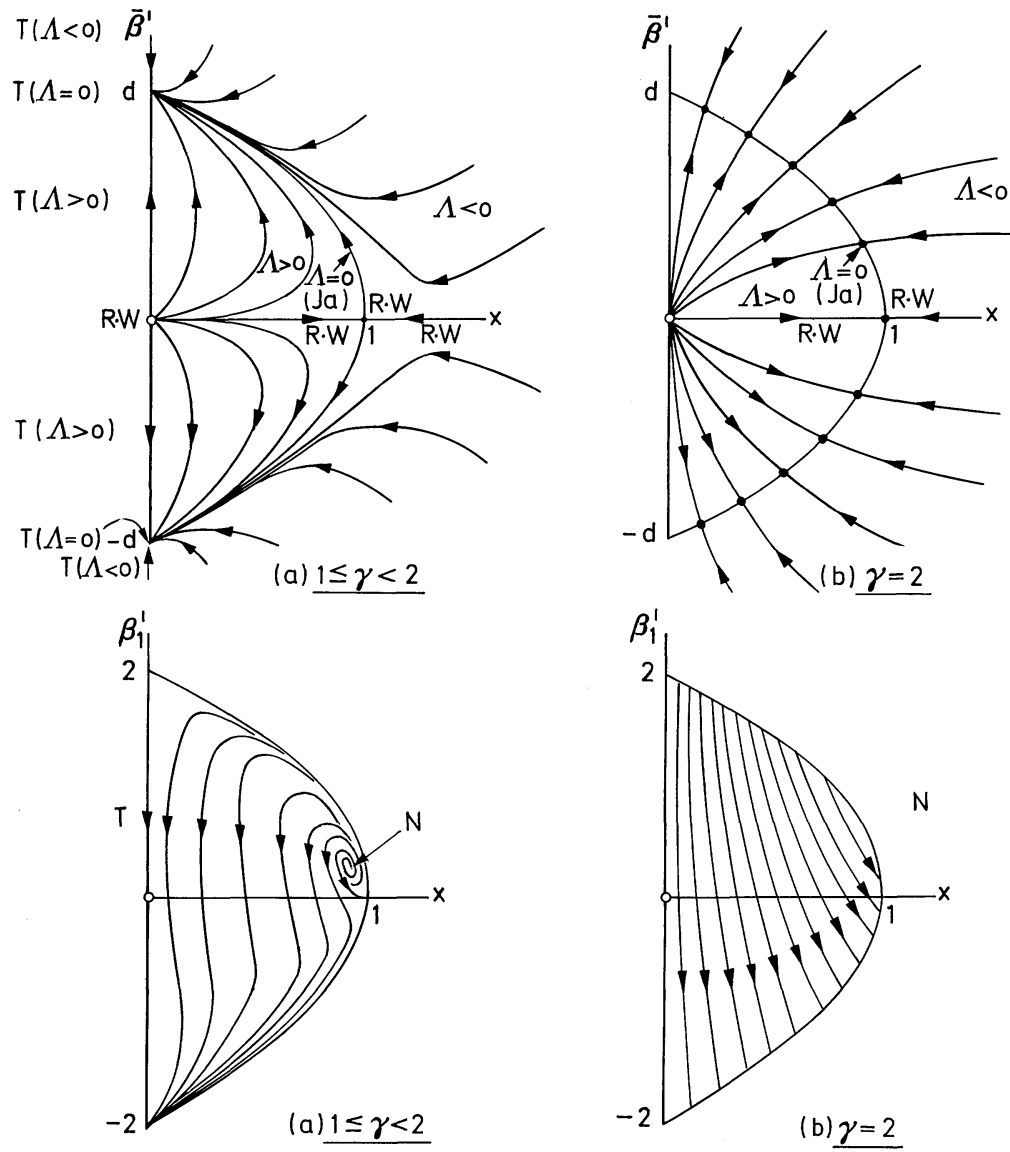

Figs. 1 and 2

Figs. 1-7 depict the evolution of a set of models (in general with zero cosmological constant) in terms of the variables $x=\frac{1}{3} \mu z$ and $\beta_{1}^{\prime}, \beta_{2}^{\prime}$ or $\beta^{\prime} . x$ is a measure of the importance of matter, and $\beta_{1}^{\prime}, \beta_{2}^{\prime}$ and $\beta^{\prime}$ measure the importance of shear anisotropy. The axes are drawn in the conventional sense (e.g. $x$ increases from left to right in Fig. 1-5, 7) but the directions are not marked, to prevent confusion with trajectories. Arrows refer to evolution in $\Omega$-time $(\Omega \rightarrow+\infty$ indicates $t \rightarrow 0$, or approach to the singularity). Diagrams (a) refer to values of the parameter $\gamma$ lying in the range $1 \leqq \gamma<2$, and (b) to $\gamma=2$. In Fig. 1, trajectories are drawn for the general Bianchi type I model with a $\Lambda$-term. Fig. 2 describes the LRS $\left(\beta_{2} \equiv 0\right)$ type II model, Fig. 3 the $\left(n_{\alpha}^{\alpha}=0\right)$ type V model (note the resemblance between the evolution in this case and the type I case with $\Lambda>0)$, Fig. 4 the axisymmetric $\left(\beta_{2} \equiv 0\right)$ type $\mathrm{VI}_{0}$ model, and Fig. 5 the $n^{\alpha}{ }_{\alpha}=0$ type $\mathrm{VI}_{h}(h \neq 0)$ model, whose behaviour differs according as (ai) $C^{2} \leqq 3 \gamma-2$ or (aii) $C^{2}>3 \gamma-2$. The general type II vacuum solution is depicted in Fig. 6, and a special type II solution with $\beta_{1}^{\prime} \equiv \frac{1}{4}(3 \gamma-2)$ in Fig. 7. New exact solutions are denoted by $N$, and those already known by $E-M, J a, J o, K, R-W$ and $T$, which refer respectively to the authors Ellis and MacCallum, Jacobs, Joseph, Kantowski and Sachs, Robertson and Walker, and Taub. 


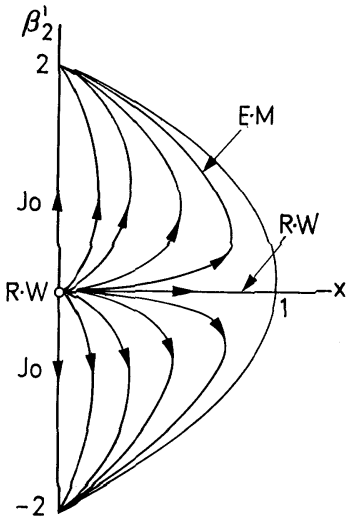

(a) $1 \leq \gamma<2$

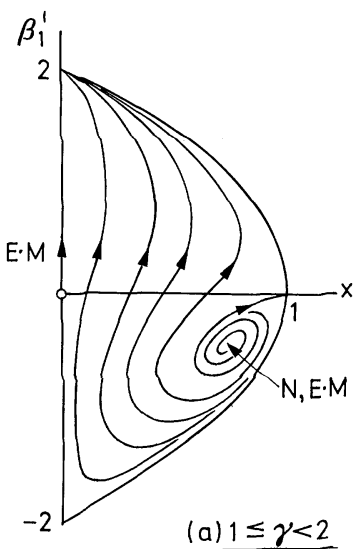

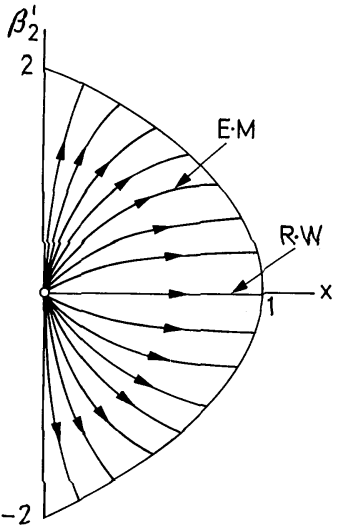

(b) $\gamma=2$

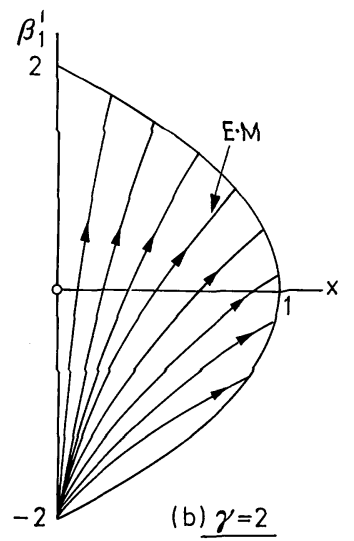

Figs. 3 and 4

referred to in [5]. The latter solution has properties similar to those of a special exact radiation solution of Kantowski [15] (see Fig. 5(a)), having a point singularity and with matter dynamically important at early times. When $\gamma=2$, matter is always dynamically important, in that $x$ is bounded away from zero at all times; the singularity is a point singularity. Further, certain of these models fail to bounce against the potential wall, their trajectories remaining in the region $\beta_{1}^{\prime}>0$ for all time (Fig. 2(b)). The equations may be integrated exactly (see appendix). All the type II models considered here are LRS [9].

The type V models $(k=0)$ of our set are all known up to a quadrature [9]. Joseph [14] has presented the empty solution, and Ellis and MacCallum [9] have determined the solutions for $\gamma=1, \frac{4}{3}, \frac{5}{3}$ and 2 in terms of elliptic functions. From Fig. 3, there are no bounces, the models 

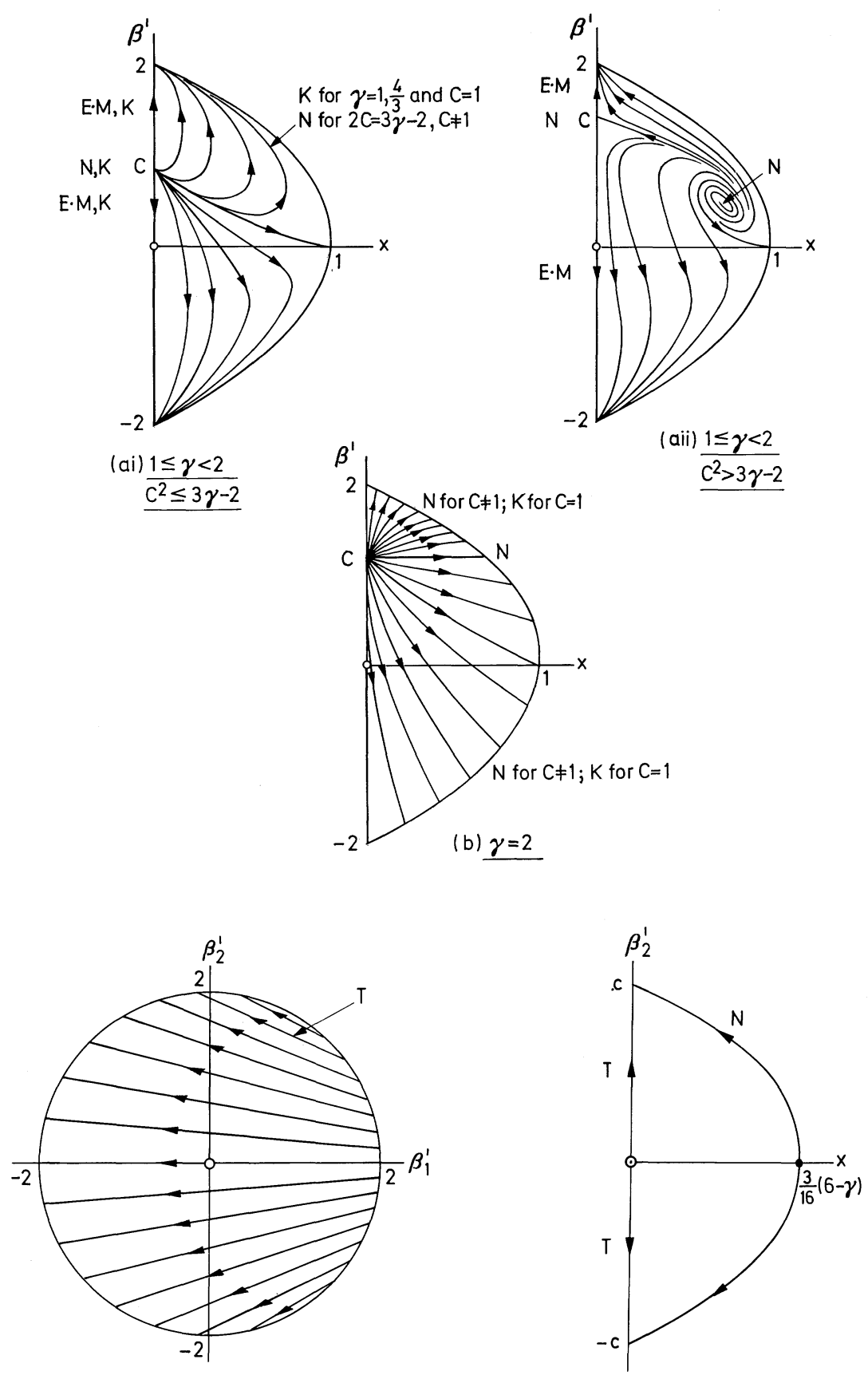

Figs. 5, 6 and 7 
isotropize ( $\beta^{\prime} \rightarrow 0$ as $\Omega \rightarrow-\infty$ ), and $x \rightarrow 0$ as $\Omega \rightarrow+\infty$ in all cases except for the Robertson-Walker (point-singularity) models and for the $\gamma=2$ solutions, and so matter is generally dynamically unimportant in the early stages. Cigar singularities, with the 2- or 3-axes preferred, occur for $1 \leqq \gamma<2$; for $\gamma=2$, we obtain barrels or cigars (with the 2- or 3-axis preferred), or points. These statements accord with those of MacCallum [20].

Ellis and MacCallum [9] have integrated the equations for axisymmetric type $\mathrm{VI}_{0}$ models which are empty or which contain matter obeying the equation of state $p=\mu$. For all models with $1 \leqq \gamma<2$, we see from Fig. 4(a) that the behaviour of the solutions is qualitatively similar to that of type II. The singularity is cigar-shaped, with the 1-axis preferred [20]. For each value of $\gamma$ two exceptional cases (both with point singularities) occur in which $x$ does not tend to zero as $\Omega \rightarrow+\infty$, and so matter effects are not negligible in these models. The special case of $x$ and $\beta_{1}^{\prime}$ identically constant can be integrated exactly, giving solutions which, except for the dust case [9], are new (see appendix). In the case when $\gamma=2$, there is also some similarity between Figs. 2(b) and 4(b); a cigar or barrel singularity, with the 1 -axis preferred, or a point singularity will occur.

New solutions (see appendix) may be obtained for those models of type $\mathrm{VI}_{h}\left(h \neq 0,-\frac{1}{9}\right)$ which contain a fluid whose equation of state is $p=\mu$. For the type $\mathrm{VI}_{h}$ models with which we can deal, we have $0<k<+\infty$, $k \neq 3(0<C<2, C \neq \sqrt{3})$ and $k=C=1$ for Bianchi type III, which is of type $\mathrm{VI}_{h}$ in the classification of Ellis and MacCallum [9]. Kantowski [15] has discussed the case $\mathrm{C}=1$, giving all solutions except that in which $\beta^{\prime} \equiv 1$, which corresponds to a formally infinite value of his parameter $a$. Matter is never negligible in the early stages, and for some solutions the sign of $\beta^{\prime}$ changes exactly once, corresponding to a collision against the potential wall. These models do not isotropize. When $\gamma<2$, the behaviour falls naturally into two cases. When $C^{2} \leqq 3 \gamma-2$ (which always holds for Bianchi type III) $x \rightarrow 0$ as $\Omega \rightarrow-\infty$ for all cases, and $x \rightarrow 0$ as $\Omega \rightarrow+\infty$ except for one case, which for $C=1$ and $\gamma=\frac{4}{3}$ corresponds to the Kantowski radiation solution referred to earlier, in which matter is initially (in $t$-time) dynamically important and in which there is a point-singularity. Some solutions bounce against the wall $\left(\beta^{\prime}\right.$ changes sign). We have obtained solutions for all cases where $2 C=3 \gamma-2$, which apart from the case $C=1, \gamma=\frac{4}{3}$ are new. Kantowski (op.cit.) has also given the vacuum solution for $C=1$, and Ellis and MacCallum [9] have extended this to the case $C \neq 1$, giving those solutions for which $\beta^{\prime}$ is not identically equal to $C$. This exceptional case is given as a new exact solution in the appendix. Kantowski and Sachs [16] have given exact solutions for the case $C=\gamma=1$. When $C^{2}>3 \gamma-2$, we obtain a 
focus, from which all non-empty solutions "start" (in $\Omega$-time). Most solutions bounce against the potential wall, and in general matter is dynamically negligible at early times. A new exact solution is given for the case where $x$ and $\beta^{\prime}$ are identically constant (cf. Types II and $\mathrm{VI}_{0}$ ), including the vacuum case $\left(\beta^{\prime} \equiv C\right)$. None of the $\mathrm{VI}_{h}(h \neq 0)$ models of our set isotropizes in $t$-time. For $k>1$ (respectively, $k<1$ ) a cigarsingularity with the 1- or 3-axis (respectively, 2- or 3-axis) preferred will occur for $\gamma<2$ [20]. For $k=1$, there is a pancake or a cigar, with the 3 -axis preferred. If $C^{2}>3 \gamma-2$, the solution at the focus can have a cigar or a barrel singularity (with the 3-axis distinguished), or a pointsingularity. When $\gamma=2$, there is a point singularity, or for $k>1$ (respectively, $k=1, k<1)$ there can be cigar or barrel singularities with the 1- or 3-axis (respectively, 3-axis, 2- or 3-axis) distinguished. The special vacuum solution $\beta^{\prime} \equiv C$ has a cigar or barrel (with the 3-axis distinguished) singularity, according as $k>1$ or $k=1$, or a point singularity (for $k<1$ ).

The Figs. 1-5 provide us with a unified view of the solutions of our set. A cursory inspection gives information concerning the qualitative nature of the solutions, in particular about asymptotic $(\Omega \rightarrow \pm \infty)$ behaviour. To what extent a solution is typical may be readily assessed. Further, the diagrams are suggestive of which solutions may next be most easily obtained in exact form: exact solutions are still unknown for those types II, $\mathrm{VI}_{0}$ and $\mathrm{VI}_{h}$ models which spiral around a focus, and if one were found it might readily lead to a solution for all cases, in view of the similarity of the behaviour in the different types. The other remaining solutions not yet solved in closed form are of type $\mathrm{VI}_{h}$ with $C \leqq V(3 \gamma-2)<2,0<C<2, C \neq \sqrt{3}, 2 C \neq 3 \gamma-2$, and because these are the only unknown solutions which do not spiral, it is likely that they will be the next to be obtained, if further exact integrations are possible.

\section{Special Subsystems}

Because the type I models have several simplifying features, we can still obtain a pictorial description of the solutions for these models when $\Lambda \neq 0$. With $\bar{\beta}$ defined as before we obtain Figs. 1(a), (b). When $\Lambda>0$, Eq. (1.6) implies $\beta^{\prime 2}+4 x<4$, and all models isotropize in $t$-time (i.e. $\beta^{\prime} \rightarrow 0$ as $\left.\Omega \rightarrow-\infty\right)$. When $1 \leqq \gamma<2$, matter effects are unimportant in the early stages, except for the Robertson-Walker model $\left(\beta^{\prime} \equiv 0, x \rightarrow 1\right.$ as $\Omega \rightarrow+\infty$ ); when $\gamma=2$ matter is important in all cases. As for all type I models, $\beta^{\prime}$ is of constant sign; there are no "bounces". The nature of the singularity is essentially independent of the $\Lambda$-term (see Fig. 1). If $\Lambda<0$, a recollapse occurs in finite time [20]. From (1.6), $\beta^{\prime 2}+4 x>4$, 
and the recollapse in our diagrams is represented by the trajectories going out (in $t$-time) to infinity, where $z=0$, in a finite time. Matter is important in the early stages only when $\gamma=2$. A discussion of certain type I models with $\Lambda \neq 0$ has been given by Stewart and Ellis [35] and by Saunders [33].

The evolution of the general $\left(\beta_{2} \neq 0\right)$ type II model with $\Lambda=0$ can also be visualised, but a three-dimensional picture is necessary. We write (1.5a) in intrinsic coordinates $(s, \psi)$, where $s^{\prime} \equiv \beta^{\prime}$ and $\psi$ is the angle that the direction of motion makes with the positive $\beta_{1}$-axis. The acceleration of a point in the $\beta$-plane which has non-zero velocity is $\boldsymbol{\beta}^{\prime \prime}=s^{\prime \prime} \boldsymbol{t}+s^{\prime} \psi^{\prime} \boldsymbol{n}$, where $\boldsymbol{t}$ and $\boldsymbol{n}$ are respectively unit tangent and normal directions to the motion. Then $\left(\beta_{1}^{\prime \prime}, \beta_{2}^{\prime \prime}\right)=\frac{s^{\prime \prime}}{s^{\prime}}\left(\beta_{1}^{\prime}, \beta_{2}^{\prime}\right)+\psi^{\prime}\left(-\beta_{2}^{\prime}, \beta_{1}^{\prime}\right)$ implies

$$
\begin{aligned}
& s^{\prime \prime}=\frac{1}{2} s^{\prime}\left\{4-(3 \gamma-2) x-s^{\prime 2}\right\}-\frac{2}{3} z e^{2 \Omega+4 \beta_{1}} \cos \psi, \\
& \psi^{\prime}=\frac{2}{3} \frac{z}{s^{\prime}} e^{2 \Omega+4 \beta_{1}} \sin \psi,
\end{aligned}
$$

with

$$
{s^{\prime 2}}^{2}=4-4 x-\frac{1}{3} z e^{2 \Omega+4 \beta_{1}}<4 .
$$

We adopt the convention that $s^{\prime} \geqq 0$ and $0 \leqq \psi<2 \pi$, and assume that $\psi$ and $\psi^{\prime}$ are continuous functions of $\Omega$ except possibly when $\psi=0$. This means that except for when the universe-point is moving directly towards the potential wall, any deflection of the point's direction takes place gradually; only when $\psi=0$ can $\psi$ change discontinuously, to $\pi$. From (1.5aii) or (3.2) we see that if $\beta_{2}^{\prime}$ is ever zero, this always holds. This corresponds to the axisymmetric (LRS) case, which has already been mentioned. From now on, we can thus assume $\psi \neq 0, \pi$. From (3.2), $s^{\prime}>0$, for if $s^{\prime}$ were zero in finite $\Omega$-time, $\psi^{\prime}$ would be infinite. Further, $\psi^{\prime}$ has the same sign as $\pi-\psi$, and so if $0<\psi<\pi$ at some time, this occurs thereafter (in $\Omega$-time), and therefore $\psi$ tends to some limit, $\psi_{0}$. If $\psi_{0}=\pi,(3.1)$ and (3.2) give

$$
\frac{s^{\prime \prime}}{s^{\prime}}=\frac{1}{2}\left\{4-(3 \gamma-2) x-{s^{\prime 2}}^{2}\right\}-\varepsilon^{\prime} \frac{\cos \varepsilon}{\sin \varepsilon}
$$

where $\psi=\pi-\varepsilon, \varepsilon>0$ and $\varepsilon \rightarrow 0$ as $\Omega \rightarrow+\infty$. Integrating, we have $\ln \left(s^{\prime} \tan \varepsilon\right)=\frac{1}{2} \int^{\Omega}\left\{4-(3 \gamma-2) x-{s^{\prime}}^{2}\right\} d \Omega+$ constant. Because $s^{\prime}$ is bounded above, $\ln \left(s^{\prime} \tan \varepsilon\right) \rightarrow-\infty$ as $\Omega \rightarrow+\infty$, yet the integrand is positive, a contradiction. 
If $\psi_{0}<\pi$, the integrand must tend to zero (in a sense to be defined) because if $\psi=\psi_{0}-\varepsilon,(3.1)$ and (3.2) give

$$
s^{\prime \prime} \sim \frac{1}{2} s^{\prime}\left\{4-(3 \gamma-2) x-s^{\prime^{2}}\right\}+\frac{\cos \psi_{0}}{\sin \psi_{0}} \varepsilon^{\prime} s^{\prime}
$$

for sufficiently small $\varepsilon$. On integration,

$$
\ln s^{\prime} \sim \frac{1}{2} \int^{\Omega}\left\{4-(3 \gamma-2) x-{s^{\prime}}^{2}\right\} d \Omega+\frac{\varepsilon \cos \psi_{0}}{\sin \psi_{0}} .
$$

Because the integrand is positive (and $s^{\prime}$ is bounded above), given any $\varepsilon>0$ there exists a time $\Omega_{0}$ such that the integrand is less than $\varepsilon$ for $\Omega>\Omega_{0}$, except possibly on a set of $\Omega$-intervals the sum of whose lengths is less than 1 (we will say that the integrand is "arbitrarily close to zero except on a set of zero measure"). When $1 \leqq \gamma<2$, this is possible only if $x$ and $s^{\prime}$ are arbitrarily close to 0 and 2, respectively, except on a set of zero measure, because of the restriction (3.3). When $\gamma=2$, (3.1)-(3.3) give

$$
s^{\prime \prime}=\frac{1}{2} \frac{s^{\prime} \psi^{\prime}}{\sin \psi}\left\{\frac{1}{2} s^{\prime}-2 \cos \psi\right\}
$$

and, since $\psi^{\prime}>0$, if $s^{\prime}-4 \cos \psi$ is ever positive, $s^{\prime \prime}>0$ and so $s^{\prime}-4 \cos \psi$ is thenceforth (in $\Omega$-time) positive. Consequently $s^{\prime}-4 \cos \psi$ is eventually one-signed, implying that $s^{\prime}$ is eventually strictly increasing, strictly decreasing, or constant. In all cases $s^{\prime}$ tends to a limit, $s_{0}^{\prime}$, as $\Omega \rightarrow+\infty$, and because $4-4 x-s^{\prime 2}$ converges to zero by the above argument, $x$ tends to a limit, $x_{0}=1-\frac{1}{4} s_{0}^{\prime 2}$, as $\Omega \rightarrow+\infty$. In common with the axisymmetric case it appears that $x_{0}$ can take arbitrary values in the interval $(0,1]$. If $\pi<\psi<2 \pi$, the same results hold, as follows by the substitution $\phi=2 \pi-\psi$.

The vacuum case, $x \equiv 0$, is particularly easy to describe. Eqs. (1.5a) and (1.6) give

$$
\frac{d \beta_{1}^{\prime}}{d \Omega}=\frac{1}{2}\left(\beta_{1}^{\prime}-4\right)\left(4-{\beta^{\prime 2}}^{2}\right)=\frac{1}{\beta_{2}^{\prime}}\left(\beta_{1}^{\prime}-4\right) \frac{d \beta_{2}^{\prime}}{d \Omega},
$$

and so $\beta_{2}^{\prime}=\kappa\left(4-\beta_{1}^{\prime}\right)$ for some constant $\kappa$. The nature of the solutions (due to Taub [36]) is depicted in Fig. 6. It is seen that $\beta_{2}^{\prime}$ is of constant sign, and that $\beta_{1}^{\prime}$ changes sign exactly once or never at all. This corresponds, in terms of the "receding potential wall" description, to the universe-point rebounding once off the wall, or having a direction of motion inclined at such a large angle to the normals of the wall that it never reaches it. No vacuum model isotropizes. The singularity is in 
general cigar-shaped with the 2- or 3-axis distinguished; the only exception is the LRS case $\left(\beta_{2}^{\prime} \equiv 0\right)$ in which there is a pancake singularity [20].

There is another special case in type II with which we can readily deal: there exist solutions in which $\beta_{2}^{\prime} \neq 0$ and $\beta_{1}^{\prime}$ is constant. From (1.5ai) and (1.6) we have, putting $\mu=M e^{3 \gamma \Omega}$ with $M$ constant,

$$
{\beta^{\prime 2}}^{2}=4-4 x-\frac{3(2-\gamma) x \beta_{1}^{\prime}}{4-\beta_{1}^{\prime}}=4-4 x-\frac{x e^{4 \beta_{1}+2 \Omega}}{M e^{3 \gamma \Omega}} .
$$

Equating coefficients of $x, \beta_{1}=\frac{1}{4}(3 \gamma-2) \Omega+\beta_{0}$, for some constant $\beta_{0}$ which satisfies $(2-\gamma)(3 \gamma-2) M=(6-\gamma) e^{4 \beta_{0}}$. Now for Bianchi type II models, we can rescale according to the scheme $\beta_{1} \rightarrow \beta_{1}+\lambda, \Omega \rightarrow \Omega-2 \lambda$, where $\lambda=2 \beta_{0} / 3 \gamma$. This preserves the scaling which has made $N_{1}=1$ (for notation, see [9], [20]), i.e. it preserves the invariant $n_{1}=e^{2 \beta_{1}+\Omega}$, and it has the effect of transforming $\beta_{0}$ to zero. The remaining Eqs. (1.1), (1.5aii) and (1.6) (which of course show that (3.4) is preserved in time) give the exact solution described in the appendix, and illustrated in Fig. 7. This solution is a generalisation of the nonempty LRS solution found earlier [5], which appears as the point $x=\frac{3}{16}(6-\gamma), \beta_{2}^{\prime}=0$ in the diagram. We note that matter effects are in general dynamically negligible $(x \rightarrow 0)$ as $\Omega \rightarrow+\infty$, and that the models do not isotropize. The model will in general have a cigar singularity with the 2- or 3-axis preferred [20], but the particular case of $\beta_{2}^{\prime}=0$ has a point singularity (Section 2).

\section{Conclusion}

By using geometric techniques, we have been able to determine the precise behaviour of a certain set of non-rotating spatially-homogeneous cosmological models. These models are filled with a perfect fluid with an equation of state $p=(\gamma-1) \mu$, where $p$ is the isotropic pressure, $\mu$ the total energy density and $\gamma$ a constant satisfying $1 \leqq \gamma \leqq 2$. The models are further restricted because, in order to employ the methods of this paper, the potential function $V_{1}$ must satisfy a relationship of the form $\frac{\partial V_{1}}{\partial \beta}=D V_{1}$ (where $D$ is a constant and $\beta$ a quantity specifying the shear (see Section 1)). The necessity of this last restriction appears to be the only serious limitation from which the qualitative treatment suffers. The diagrams that we have obtained may be easily interpreted and the various patterns of evolution assessed. In particular, we have determined the asymptotic (towards or away from the singularity) behaviour of the variables occurring in the field equations, and thus the degree of an- 
isotropy or the dynamical importance of matter in the early or late stages of expansion of the models.

We have presented in a regularised form the field equations for a large family of cosmological models (Class A and Class B $\left(n_{\alpha}^{\alpha}=0\right)$ ). The limitations implicit in the procedure of regularisation have been discussed, and suggestions have been made of ways of avoiding these difficulties.

It is quite possible that a combination of the two methods discussed in this paper will prove useful: by applying geometric techniques to regularised systems of the field equations, detailed information concerning the behaviour of the solutions could then be obtained.

Acknowledgements. I should like to express my gratitude to Drs. G. F. R. Ellis, F. G. Friedlander, S. W. Hawking, M. A. H. MacCallum and J. M. Stewart for their useful comments and encouragement throughout the course of this work. In particular, I thank Drs. G.F.R. Ellis and M. A. H. MacCallum for several valuable suggestions concerning the presentation, and Drs. K. C. Jacobs and M.A.H. MacCallum for independently suggesting that the initial results on exact solutions of type II be extended to type VI models. I thank also the Science Research Council for a research studentship held while the work was in progress.

\section{Appendix of New Exact Solutions}

If $\gamma=2$, the equations of Section 1 may be solved simultaneously for the models admitting groups of type II and type $\mathrm{VI}_{h}(h \neq 0)$, for which exact solutions were hitherto unknown. We note that $x=K\left(\beta^{\prime}-C\right)^{2}$, where $K$ is constant, is a first integral of the system of equations. Substitution of this into the $\beta^{\prime}$-equation yields a first-order ordinary differential equation for $\beta^{\prime}$, whose solution is

$$
\beta^{\prime}=C+\frac{4-C^{2}}{C\{1+B \operatorname{coth}[a(\beta-C \Omega)]\}},
$$

where

$$
a=\frac{B C}{2}= \pm \frac{C}{2}\left[1+\frac{(4 K+1)\left(4-C^{2}\right)}{C^{2}}\right]^{1 / 2},
$$

unless $B=0$ in which case $\beta^{\prime}=C+A$, where

$$
A\left[(4 K+1) A^{2}+2 A C-\left(4-C^{2}\right)\right]=0 .
$$

If $B \neq 0$, further integration yields

$$
e^{(\beta-C \Omega)} \sinh ^{2 / C}[a(\beta-C \Omega)]=E e^{\left(4-C^{2}\right) \Omega / C}
$$


where $E$ is constant. Also $x=K\left(\beta^{\prime}-C\right)^{2}=\frac{1}{3} \frac{M e^{6 \Omega}}{\dot{\Omega}^{2}}$ implies $\dot{\beta}-C \dot{\Omega}= \pm b e^{3 \Omega}$, where $\mu=M e^{6 \Omega}$ and $b=\left(\frac{M}{3 K}\right)^{1 / 2}$. Putting $C=1$, we regain the solution of Kantowski [15]. If we let $u=\beta-C \Omega$, the equation

$$
\dot{u}= \pm \frac{b}{E^{3 C /\left(4-C^{2}\right)}} e^{3 C u /\left(4-C^{2}\right)}(\sinh a u)^{\left.6 / 4-C^{2}\right)}
$$

gives $t=t(u)$. From the above results we then obtain $\Omega=\Omega(u)$ and $\beta=u+C \Omega(u)$. If $\beta^{\prime}$ is constant $(B=0)$, so also is $x$. This implies either $x=0$ or $4-4 x-\beta^{\prime 2}=0$, both of which lead to contradictions.

We now quote all new exact solutions obtained, using the coordinate systems of [9].

1. Type II (a) $1 \leqq \gamma<2$.

$$
\begin{aligned}
& \beta_{1}=\frac{1}{4}(3 \gamma-2) \Omega, \beta_{2}^{\prime 2}=\frac{3}{16}(2+\gamma)(10-3 \gamma) \frac{E^{2} e^{3(2-\gamma) \Omega}}{1+E^{2} e^{3(2-\gamma) \Omega}}, E \geqq 0, \\
& \beta_{2}=\int_{2}^{\Omega} \beta_{2}^{\prime} d \Omega, \mu=\frac{(6-\gamma) e^{3 \gamma \Omega}}{(2-\gamma)(3 \gamma-2)}, \dot{\Omega}^{2}=\frac{16}{9} \cdot \frac{e^{3 \gamma \Omega}+E^{2} e^{6 \Omega}}{(2-\gamma)(3 \gamma-2)}, \dot{\Omega}<0, \\
& t=-\int_{\Omega}^{+\infty} \frac{d \Omega}{\dot{\Omega}} .
\end{aligned}
$$

The metric is

$$
d s^{2}=-d t^{2}+e^{-2 \Omega+2 \beta_{1}}(d x+z d y)^{2}+e^{-2 \Omega-\beta_{1}}\left(e^{\sqrt{3} \beta_{2}} d y^{2}+e^{-\sqrt{3} \beta_{2}} d z^{2}\right) .
$$

(b) $\gamma=2$.

$$
\begin{aligned}
& \beta^{2}=0, t= \pm \int^{u} \frac{e^{u}(\sinh a u)^{1 / 2}}{b E} d u, e^{-3 \Omega}=b \int_{0}^{t}\left(1+\frac{1}{2} a \operatorname{coth} a u\right) d t \\
& \beta_{1}^{\prime}=4-\frac{3}{1+\frac{1}{2} a \operatorname{coth} a u}, \beta_{1}=\int_{0}^{\Omega} \beta_{1}^{\prime} d \Omega, \mu=3 K b^{2} e^{6 \Omega} .
\end{aligned}
$$

The metric is as in 1(a).

2. Type $V_{0}$ (a) $1 \leqq \gamma<2$. $\beta_{1}^{\prime}=-\frac{1}{2}(3 \gamma-2)$. We may rescale according to $\beta_{1} \rightarrow \beta_{1}+\lambda, \Omega \rightarrow \Omega+\lambda$, which leaves invariant $n_{2}=-n_{3}=e^{\Omega-\beta_{1}}$; without loss of generality we can therefore assume $\beta_{1}=-\frac{1}{2}(3 \gamma-2) \Omega$. We obtain $\beta_{2}=0, e^{-\frac{3 \gamma}{2} \Omega}=\frac{2 \gamma t}{V\{(2-\gamma)(3 \gamma-2)\}}$ and $\mu=\frac{4 e^{3 \gamma \Omega}}{3 \gamma-2}$; the 
metric is

$$
\begin{aligned}
& d s^{2}=-d t^{2}+\left(e^{-3 \gamma \Omega}+z^{2} e^{-\frac{3}{2}(2-\gamma) \Omega}\right) d x^{2}-2 z e^{-\frac{3}{2}(2-\gamma) \Omega} \cosh x d x d y \\
& +e^{-\frac{3}{2}(2-\gamma) \Omega}\left(1+2 \sinh ^{2} x\right) d y^{2}+2 e^{-\frac{3}{2}(2-\gamma) \Omega} \sinh x d y d z+e^{-\frac{3}{2}(2-\gamma) \Omega} d z^{2} .
\end{aligned}
$$

3. Type $V I_{h}$ (a) $1 \leqq \gamma<2$.

(i) $\mu \neq 0, C \beta=(3 \gamma-2) \Omega+\lambda, \lambda$ constant, $\beta_{1}=\frac{k}{\sqrt{3}} \beta_{2}=-\frac{k \beta}{V\left(3+k^{2}\right)}$,

$$
0<k<+\infty, k \neq 3, C=\frac{2 k}{\sqrt{ }\left(3+k^{2}\right)}, \mu=\frac{A e^{\lambda}}{(2-\gamma)(3 \gamma-2)}\left[C^{2}-(3 \gamma-2)\right] \text {, }
$$

$$
A=\frac{4}{3} a_{0}^{2}\left(3+k^{2}\right), C^{2}>3 \gamma-2
$$

and

$$
e^{-\frac{3 \gamma}{2} \Omega}=2 \frac{\gamma k a_{0} e^{\lambda / 2} t}{V\{(2-\gamma)(3 \gamma-2)\}}
$$

The metric for the space-time is

$$
d s^{2}=-d t^{2}+X^{2}(t) d x^{2}+Y^{2}(t) e^{-2 a_{0}(1+k) x} d y^{2}+Z^{2}(t) e^{-2 a_{0}(k-1)} d z^{2},
$$

where

$$
X=e^{-\Omega+\beta_{1}}, Y=e^{-\Omega-\frac{\beta_{1}}{2}+\frac{\sqrt{3}}{2} \beta_{2}} \text { and } Z=e^{-\Omega-\frac{\beta_{1}}{2}-\frac{\sqrt{3}}{2} \beta_{2}} \cdot A
$$

and $a_{0}$ are constants.

(ii) $\mu \neq 0,2 C=3 \gamma-2$. Let $v=e^{-\Omega}, \lambda^{2}=e^{\beta-2 \Omega}$. Then $\mu=M e^{3 \gamma \Omega}$,

$$
t= \pm\left(\frac{12 E}{M}\right)^{1 / 2} \int^{\lambda} \frac{v^{3(\gamma-1)} d \lambda}{\lambda^{\frac{1}{2}(3 \gamma-4)}}
$$

and $v=v(\lambda)$ is given by

$$
\lambda^{-\frac{3}{2}(2-\gamma)} v^{3(2-\gamma)}-4 E \lambda^{\frac{3}{2}(2-\gamma)}-\frac{12 E}{M} \frac{(2-\gamma)}{(3 \gamma+2)} \lambda^{\frac{1}{2}(3 \gamma+2)}=F
$$

where $E$ and $M$ are positive constants, and $F$ is constant. When $\gamma=\frac{4}{3}$, the solution agrees with that of Kantowski [15]. The metric is as in 3(ai).

$$
\begin{gathered}
\text { (iii) } \mu=0, \beta^{\prime} \equiv C, \beta_{1}=\frac{k}{\sqrt{3}} \beta_{2}=-\frac{k \beta}{\sqrt{\left(3+k^{2}\right)}}, 0<k<+\infty(k \neq 1,3), \\
C=\frac{2 k}{\sqrt{\left(3+k^{2}\right)}}, \beta=C \Omega+\lambda, \lambda \text { constant. }
\end{gathered}
$$


Also,

$$
e^{-\frac{1}{2}\left(C^{2}+2\right) \Omega}=\frac{1}{2}\left(C^{2}+2\right)\left[\frac{4 a_{0}^{2}\left(3+k^{2}\right)}{3\left(4-C^{2}\right)}\right]^{1 / 2} e^{C \lambda / 2} t,
$$

and the metric is as described in 3(ai).

(b) $\gamma=2$. The solution assumes the complicated form mentioned at the beginning of this appendix. $\mu=3 K b^{2} e^{6 \Omega}$ and the metric is as described in 3(ai). The case of $C=1$ has been discovered by Kantowski [15]. There is also the special solution for which $\beta^{\prime} \equiv C$.

Although all vacuum metrics are known for the models of Section 1, we now rederive these solutions from the equations of that section, in order to exemplify the unification of our treatment. In general, the equation for $\beta^{\prime}$ can be integrated once to give

$$
\beta^{\prime}=2-\frac{4}{1+B e^{2(\beta-C \Omega)}}(B \text { constant }) .
$$

The change of variable $v=e^{\beta-c \Omega}$ enables this equation to be integrated to give

$$
E e^{\beta+2 \Omega}=\left[(2-C) B e^{\beta-C \Omega}-(2+C)\right]^{4 /(2-C)}(E \text { constant }),
$$

which is equivalent to $\beta=\beta(\Omega)$. Eq. (1.6) now implies an equation for $\dot{\Omega}$ in terms of $\Omega$ and $\beta(\Omega)$, and this yields the relation between $\Omega$ and $t$.

\section{References}

1. Arenstorf, R. F.: J. Differential Equations 6, 420 (1969).

2. Bendixson, I.: Acta Math. 24, 1 (1901).

3. Boutroux, P.: Leçons sur les functions définies par les équations différentielles $d u$ premier ordre. Paris: Gauthier-Villars 1908.

4. Carswell, R. F.: Monthly Notices Roy. Astron. Soc. 144, 279 (1969).

5. Collins, C.B., Stewart,J. M.: Monthly Notices Roy. Astron. Soc. 153, 419 (1971).

6. Dulac, H.: C. R. Acad. Sci. Paris 204, 1703 (1937).

7. Ellis, G.F.R.: J. Math. Phys. 8 , 1171 (1967).

8. - General relativity and cosmology, XLVII Corso. New York: Academic Press Inc. 1971.

9. - MacCallum, M.A.H.: Commun. math. Phys. 12, 108 (1969).

10. Hardy, G. H.: Proc. London Math. Soc. (2) 10, 451 (1912).

11. Harrison,E. R.: Astrophys. J. 142, 1643 (1965).

12. Jacobs, K. C.: Astrophys. J. 153, 661 (1968).

13. - Hughston, L. P.: Private communication (1970).

14. Joseph, V.: Proc. Cambridge Philos. Soc. 62, 87 (1966).

15. Kantowski, R.: Ph. D. thesis, University of Texas (1966).

16. - Sachs, R. K.: J. Math. Phys. 7, 443 (1966).

17. Levi-Civita, T.: Acta Math. 30, 305 (1904). 
18. Levi-Civita, T.: Verh. des III. Int. Math. Kongr. 1904, p. 402 (1905).

19. MacCallum, M.A.H., Ellis, G.F.R.: Commun. math. Phys. 19, 31 (1970).

20. - Commun. math. Phys. 20, 57 (1971).

21. Markus, L.: Contributions to the Theory of Nonlinear Oscillations. Vol. V, ed. L. Cesari, J. LaSalle and S. Lefshetz. Princeton: Princeton University Press 1960.

22. Matzner, R. A., Shepley,L.C., Warren, J. B.: Ann. Physics 57, 401 (1970).

23. Misner, C. W.: Astrophys. J. 151, 431 (1968).

24. - Phys. Rev. Letters 22, 1071 (1969).

25. - Phys. Rev. 186, 1319 (1969).

26. - Relativity, ed. M. Carmeli, S. I. Fickler and L. Witten. New York and London: Plenum Press 1970.

27. Nemytskii, V.V., Stepanov,V.V.: Qualitative Theory of Differential Equations. Princeton: Princeton University Press 1960.

28. Okerson, D.J.: Reported in Misner (1970).

29. Oszvath, I.: J. Math. Phys. 11, 2860 (1970).

30. Rhoades, C. E., Jr., Ruffini, R.: Astrophys. J. 163, L 83 (1971).

31. Ryan, M.: Ph. D. thesis, University of Maryland, Tech. Rpt. No. 70-046 (1970).

32. Sansone, G., Conti,R.: Non-Linear Differential Equations, translated from the Italian by A.H. Diamond. Oxford: Pergamon Press 1964.

33. Saunders, P.T.: Ph.D. thesis, King's College, London University (1967).

34. Sell, G.R.: Differential Equations and Dynamical Systems, ed. J. K. Hale and J. P. LaSalle. New York and London: Academic Press 1967.

35. Stewart, J. M., Ellis, G.F.R.: J. Math. Phys. 9, 1072 (1968).

36. Taub, A. H.: Ann. of Math. 53, 472 (1951).

37. Vojtasek, S., Janac, K.: Solution of Non-Linear Systems, translated by P. Dolan. London: Iliffe Books Ltd., 1969.

38. Waldvogel, J.: Bull. Astronom. 2, 295 (1967).

39. Zel'dovich, Ya.B.: Z.E.T.F. 41, 1609 (1961), translated in Soviet Physics JETP. 14, 1143 (1962).

C. B. Collins

Dept. of Applied Mathematics

and Theoretical Physics

Silver Street

Cambridge CB 39 EW, England 